\title{
First results of Chinese particle instruments in the Double Star Program
}

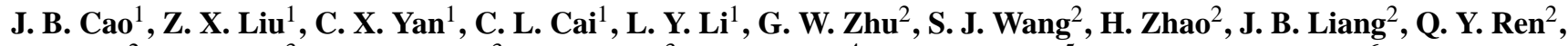 \\ Y. Y. Zai ${ }^{2}$, H. R'eme ${ }^{3}$, I. Dandouras ${ }^{3}$, C. Aoustin ${ }^{3}$, P. Escoubet ${ }^{4}$, A. Fazakerley ${ }^{5}$, S. McKenna-Lawlor ${ }^{6}$, and \\ M. Dunlop ${ }^{7}$ \\ ${ }^{1}$ Key laboratory for space weather, CSSAR, Beijing, 100080, China \\ ${ }^{2}$ Center for Space Science and Applied Research, Beijing, 100080, China \\ ${ }^{3}$ Centre d'Etude Spatiale des Rayonnements, 31028, Toulouse, France \\ ${ }^{4}$ ESA/ESTEC, Noordwijk, The Netherlands \\ ${ }^{5}$ Mullard Space Science Laboratory, Surrey, UK \\ ${ }^{6}$ STIL, National University of Ireland, Maynooth, Ireland \\ ${ }^{7}$ The Blackett Laboratory, Imperial College, London, UK
}

Received: 15 February 2005 - Revised: 9 May 2005 - Accepted: 20 June 2005 - Published: 8 November 2005

Part of Special Issue "Double Star - First Results"

\begin{abstract}
Double Star Program (DSP) aims to investigate the trigger mechanism and physical models of geomagnetic storms and substorms. The DSP involves two satellites: the equatorial satellite of DSP (TC-1 in China) and the polar satellite of DSP (TC-2 in China). On board the two satellites of TC- 1 and TC-2, there are four kinds of particle instruments developed by the Center for Space Science and Applied Research (CSSAR), namely: the High Energy Electron Detector (HEED, TC-1, 2), the High Energy Proton Detector (HEPD, TC-1, 2), the High Energy Heavy Ion Detector (HID, TC-1, 2) and the Low Energy Ion Detector (LEID, TC2). HEED, HEPD and HID were developed and calibrated in China. The LEID was developed in China and calibrated in France. This paper introduces the scientific objectives and characteristics of each instrument, their status and some preliminary results.
\end{abstract}

Keywords. Magnetospheric Physics (Instruments and techniques; Storms and substorms; Energetic particles, trapped)

\section{Introduction}

Double Star Program (DSP) is the first joint endeavour between the China National Space Administration (CNSA) and the European Space Agency (ESA). The DSP consists of two satellites: the equatorial satellite of DSP (TC-1 in China) and the polar satellite of DSP (TC-2 in China). The TC-1 is at an eccentric equatorial orbit with an apogee of about 13.4 $R_{E}$ and the TC-2 is at a polar orbit with an apogee of

Correspondence to: J. B. Cao

(jbcao@ center.cssar.ac.c) about 6.0 $R_{E}$. The equatorial satellite of DSP with on-board particle and field instruments will detect the physical processes of magnetic storms and magnetospheric substorms in the near-Earth magnetotail, as well as the energy transfer from the solar wind to the magnetosphere via the dayside magnetopause. The polar satellite of DSP, capable of making remote sensing observations as well, will detect energy transfer from the solar wind and the near-Earth magnetotail to the polar ionosphere and upper atmosphere, as well as ionized particle transfer from the ionosphere to the magnetosphere.

The main goal of DSP is to investigate the trigger mechanism and physical models of geomagnetic storms and substorms. The geospace environment comprises magnetic and electric fields, as well as particles of various energies and species. During geomagnetic storms and magnetospheric substorms, the behaviour of particles in the magnetosphere changes abruptly. For example, during a substorm, particle precipitation is greatly enhanced and produces strong auroral activity. During magnetic storms, a ring current can form and the high energy electron flux in the inner magnetosphere becomes significantly enhanced (Baker et al., 1998; Li et al., 1999; Zhou et al., 2001; Cao et al., 2002; Li et al., 2005a). So the scientific objectives of DSP require the ability to make observations of particles of different species and energies.

CSSAR is responsible for providing the high energy particle detectors (HEED, HEPD and HID on board both TC-1 and 2), as well as the Low Energy Ion Detector (LEID on board TC-2). Besides these particle instruments, CSSAR also provides the wave detector LFEW (Low Frequency Electromagnetic Wave detector) for TC-2. Figures 1 and 2 show pictures of the flight model of HEPD on board TC-1 and the flight model of LEID on board TC-2, respectively. 


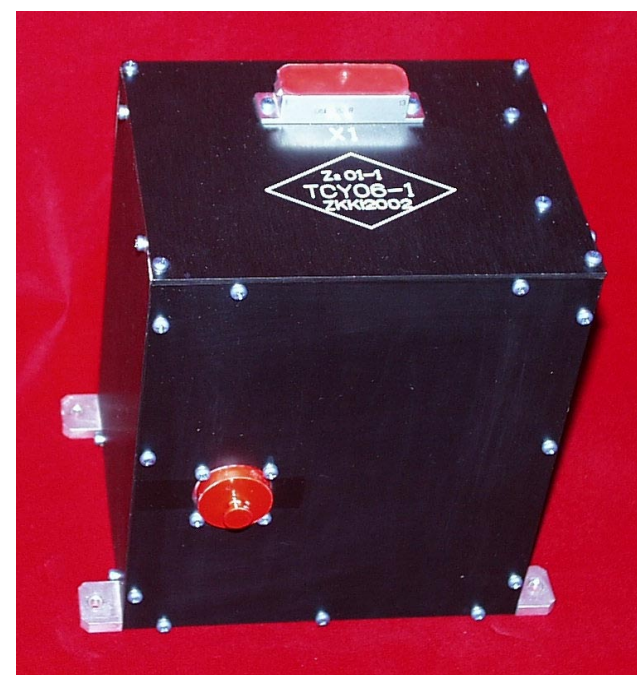

Fig. 1. Flight model of HEPD on board of TC-1.

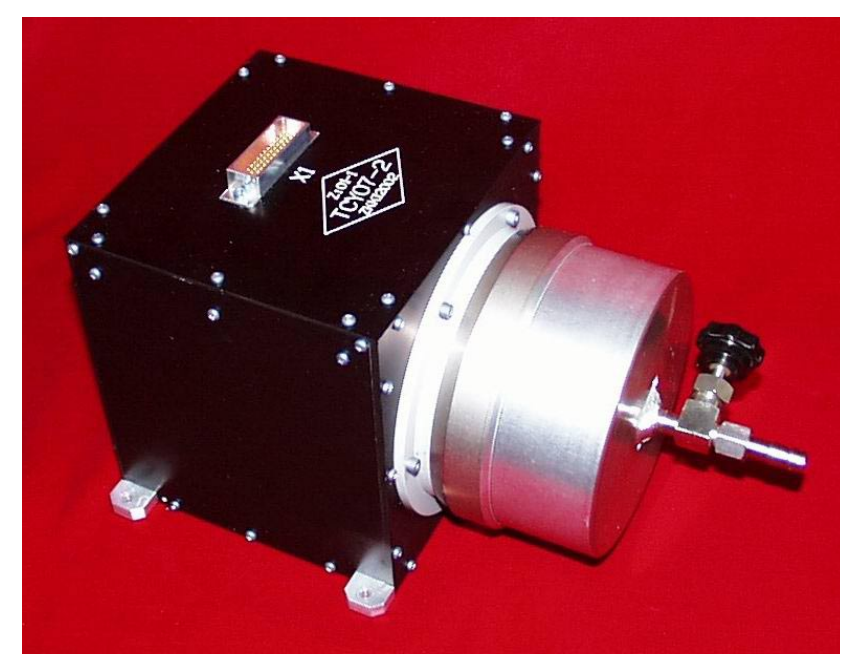

Fig. 2. Flight model of LEID on board of TC-2.

\section{Instrument descriptions}

\subsection{Technical descriptions of HEED, HEPD and HID}

There are three Chinese high-energy particle instruments on board both TC-1 and TC-2:

- The High Energy Electron Detectors (HEED) measure high energy electrons in the range of $200 \mathrm{keV}$ to $10 \mathrm{MeV}$.

- The High Energy Proton Detectors (HEPD) measure high energy protons in the energy range $3 \mathrm{MeV}$ to $400 \mathrm{MeV}$.

- The Hot Ion Detectors (HID) measure high energy heavy ions in the energy range $10 \mathrm{MeV}(\mathrm{He})$ to $8 \mathrm{GeV}(\mathrm{Fe})$ with atomic numbers from $2(\mathrm{He})$ to $26(\mathrm{Fe})$.

The detailed design of these Chinese particle instruments can be found in Zhu et al. (2002). Inside each instrument of HEED, HEPD and HID, there are three solid-state detectors (SSD): D1, D2 and D3. Energy loss measurements in these

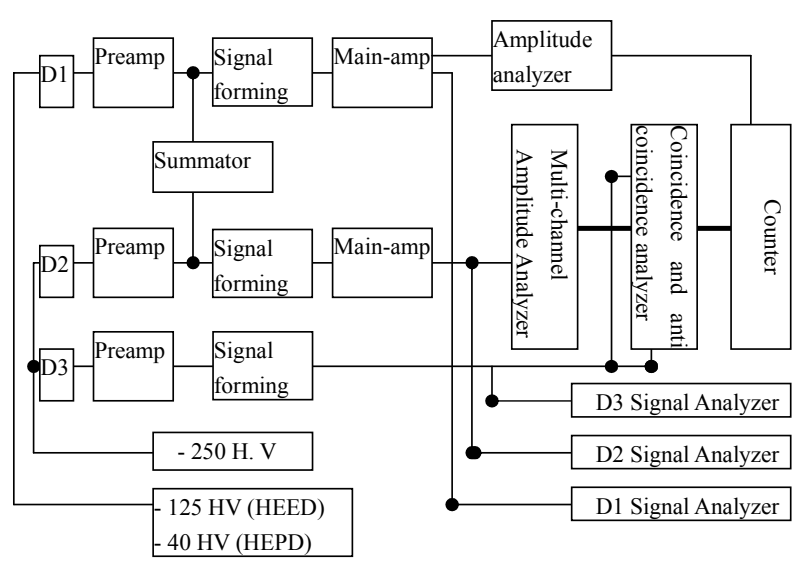

Fig. 3. Block diagrams of HEED and HEPD.

three SSDs make it possible to separate electrons, protons, and heavy ions.

A set of amplitude and coincidence-logic based on different thresholds enables determinations of particle identification and energy. The coincidence and anti-coincidence analyzer allows for a separation of electrons from protons.

Figure 3 shows the common block diagram of HEED and HEPD. Although the block diagrams of HEED and HEPD are similar, their software and thresholds are different.

HEED has 9 energy channels and HEPD has 8 energy channels. Table 1 shows the determination criteria of electrons of HEED and protons of HEPD. The subscript indicates the thresholds of energy deposited in the SSD in the unit $\mathrm{MeV}$. The symbol $\overline{E n}$ means that the counts in the $\mathrm{n}$ energy channel should be removed.

Figure 4 shows the block diagram of HID. Since the dynamic range of signals from the SSD is too large, two additional logarithmic amplifiers are used for HID.

HEED and HEPD have an opening angle of $30^{\circ}$. Since the focus of the design of HID is to measure cosmic rays, that is, particles of very low abundance, HID has a larger opening angle $\left(60^{\circ}\right)$ and correspondingly, a large geometric factor.

\subsection{HEED}

The main scientific objectives of HEED are as follows:

- To study spatial and temporal variations in the distribution of high energy electrons in the radiation belts during magnetospheric substorms and magnetic storms;

- To study the acceleration mechanism affecting high energy electrons in the near-Earth magnetosphere during CMEs (Coronal Mass Ejection);

- To study the precipitation processes of the high energy electrons, as well as the altitude and latitude distribution of their fluxes during magnetospheric substorms and magnetic storms;

- To study the spatial and temporal variation of the highenergy electrons in the near- Earth plasma sheet, as well as 
Table 1. Determination criteria of electrons of HEED and protons of HEPD.

\begin{tabular}{|c|c|c|}
\hline & HEED & HEPD \\
\hline E0 & $D 1_{0.2} \cdot \overline{D 1_{0.31}}$ & $\mathrm{P} 1 \quad(D 1+D 2)_{2.65} \cdot D 1 \cdot \overline{P 2}$ \\
\hline E1 & $(D 2+D 3)_{0.194} \cdot D 1_{0.125} \cdot \overline{D 1}_{0.31} \cdot \overline{E 2}$ & $\mathrm{P} 2 \quad(D 1 £ \ll D 2)_{4.76} \cdot D 1 \cdot \overline{P 3}$ \\
\hline E2 & $(D 2+D 3)_{0.313} \cdot D 1_{0.125} \cdot \overline{D 1_{0.31}} \cdot \overline{E 3}$ & P3 $(D 1 £ \ll D 2)_{9.86} \cdot D 1 \cdot \overline{P 4}$ \\
\hline E3 & $(D 2+D 3)_{0.432} \cdot D 1_{0.125} \cdot \overline{D 1_{0.31}} \cdot \overline{E 4}$ & P4 $\quad(D 1 £ \ll D 2)_{19.92} \cdot D 1 \cdot \overline{D 1+D 2_{33}}$ \\
\hline E4 & $(D 2+D 3)_{0.633} \cdot D 1_{0.125} \cdot \overline{D 1_{0.31}} \cdot \overline{(D 2+D 3)}$ & P5 $\quad(D 1 £ \ll D 2)_{13.8} \cdot D 3 \cdot \overline{(D 1+D 2)_{33}}$ \\
\hline E5 & $(D 2+D 3)_{0.835} \cdot D 1 \cdot \overline{D 1} \cdot \overline{E 6}$ & P6 $(D 1 £ \ll D 2)_{7.5} \cdot D 3 \cdot \overline{P 5}$ \\
\hline E6 & $(D 2+D 3)_{1.34} \cdot D 1 \cdot \overline{D 1} \cdot \overline{E 7}$ & $\mathrm{P} 7 \quad(D 1 £ \ll D 2)_{4.6} \cdot \overline{D 3} \cdot \overline{P 6}$ \\
\hline E7 & $(D 2+D 3)_{1.83} \cdot D 1 \cdot \overline{D 1} \cdot \overline{E 8}$ & P8 $\quad(D 1 £ \ll D 2)_{3.1} \cdot D 3 \cdot \overline{P 7}$ \\
\hline E8 & $(D 2+D 3)_{2.83} \cdot D 1 \cdot \overline{D 1} \cdot \overline{(D 2+D 3)}$ & \\
\hline
\end{tabular}

their injection towards the radiation belt and ring current regions.

The instrument data summary of HEED is given in Table 2.

\subsection{HEPD}

The main scientific objectives of HEPD are as follows:

- To study the energy spectrum and flux of solar cosmic rays (solar proton events) and galactic cosmic rays in the near-Earth magnetosphere;

- To study the temporal and spatial variations of high energy protons in the radiation belt during magnetospheric substorms and magnetic storms;

- To study variations in the radiation belts during the period of solar proton events;

- To study the transport processes of solar protons from the cleft, polar cap and auroral zone into the ionosphere, and in particular the resulting influence on the ionosphere in the polar region.

The instrument data summary of HEPD is given in Table 3.

\subsection{HID}

The main scientific objectives of HID are as follows:

- To measure and study the species, energy spectrum and flux of heavy ions of galactic cosmic rays;

- To measure and study solar cosmic rays in the radiation belts;

- To measure the species of heavy ions having medium and high energies in the magnetosphere.

The instrument data summary of HID is given in Table 4.

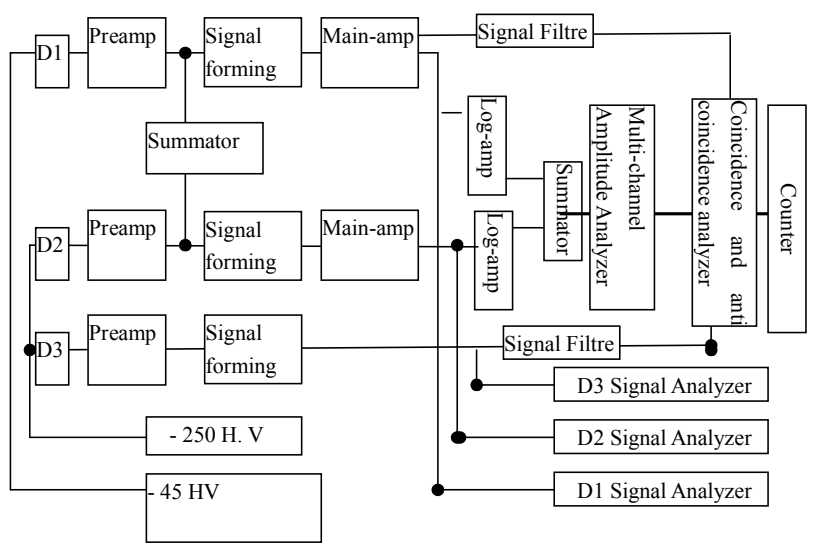

Fig. 4. Block diagram of HID.

\subsection{LEID}

The instrument LEID is mounted only on the polar orbit satellite (TC-2) and is used to measure energy spectrum, the 3-D velocity distribution function and the differential flux of low energy ions. Figure 5 shows the block diagram of LEID. The working principle and hardware design of LEID are almost identical to that of HIA on Cluster II (Rème et al., 1997). However, the software of LEID is different from that of HIA. On board TC-2, LEID does not perform any processing (for example, the moment calculation) of the raw data, and the complete ion distribution functions are sent into the telemetry. Thus, the time resolution of LEID is only $12 \mathrm{~s}$ (3 times the satellite spin period) due to the telemetry limitation. LEID only produces the 3 -D velocity distribution function for the first spin period of each 12-s interval. 
Table 2. Instrument data summary of HEED.

\begin{tabular}{|c|c|}
\hline Quantity & Value \\
\hline Mass & $2.0 \mathrm{~kg}$ \\
\hline Power & $1.7 \mathrm{~W}$ \\
\hline Geometric Factor & $0.0274 \mathrm{~cm}^{2} \mathrm{sr}$ \\
\hline Count range & 0 to $10^{5}$ per second \\
\hline Opening Angle (full width) & $\begin{array}{l}30^{\circ} \text {, circular, with a pointing direction } \\
\text { perpendicular to the spin axis of satellite }\end{array}$ \\
\hline Energy range & $200 \mathrm{keV}-10 \mathrm{MeV}$ \\
\hline Scientific telemetry rate & $0.32 \mathrm{kbit} / \mathrm{s}$ \\
\hline Temperature & $+5^{\circ} \mathrm{C}$ to $+25^{\circ} \mathrm{C}$ \\
\hline Size & $150 \times 120 \times 170 \mathrm{~mm}$ \\
\hline 9 energy channels & $\begin{array}{l}\text { E0: } 0.2-0.4 \mathrm{MeV} \text {, E1: } 0.4-0.5 \mathrm{MeV} \text {, } \\
\text { E2: } 0.5-0.6 \mathrm{MeV} \text {, E3: } 0.6-0.8 \mathrm{MeV} \text {, } \\
\text { E4: } 0.8-1.0 \mathrm{MeV} \text {, E5: } 1.0-1.5 \mathrm{MeV}, \\
\text { E6: } 1.5-2.0 \mathrm{MeV} \text {, E7: } 2.0-3.0 \mathrm{MeV}, \\
\text { E8: } 3.0-10.0 \mathrm{MeV}\end{array}$ \\
\hline 1 environment monitoring channel & $\begin{array}{l}30-400 \mathrm{MeV} \text {, downlinked as housekeeping } \\
\text { parameters }\end{array}$ \\
\hline Sampling time & $0.33 \mathrm{~s}$ \\
\hline Operation mode & continuous working mode \\
\hline Time resolution & $0.33 \mathrm{~s}$ for 9 energy channels \\
\hline & $19.8 \mathrm{~s}$ for environment monitoring channel \\
\hline
\end{tabular}

Table 3. Instrument data summary of HEPD.

\begin{tabular}{ll}
\hline \multicolumn{1}{c}{ Quantity } & \multicolumn{1}{c}{ Value } \\
\hline Mass & $2.0 \mathrm{~kg}$ \\
Power & $1.7 \mathrm{~W}$ \\
Geometric Factor & $0.1105 \mathrm{~cm}^{2} \mathrm{sr}$ \\
Count range & 0 to $10^{5}$ per second \\
Opening Angle (full width) & $30^{\circ}$, circular, with a pointing direction \\
& perpendicular to the spin axis of satellite \\
Energy range: & $3-400 \mathrm{MeV}$ \\
Scientific telemetry rate & $0.32 \mathrm{kbit} / \mathrm{s}$ \\
Temperature & $+5^{\circ} \mathrm{C}$ to $+25^{\circ} \mathrm{C}$ \\
Size & $150 \times 120 \times 170 \mathrm{~mm}$ \\
8 energy channels & $\mathrm{P} 1: 3.0-5.0 \mathrm{MeV}$ \\
& $\mathrm{P} 2: 5.0-10.0 \mathrm{MeV}$ \\
& $\mathrm{P} 3: 10.0-20.0 \mathrm{MeV}$ \\
& $\mathrm{P} 4: 20-30 \mathrm{MeV}$ \\
& $\mathrm{P} 5: 30-50 \mathrm{MeV}$ \\
& $\mathrm{P} 6: 50-100 \mathrm{MeV}$ \\
Time resolution & $\mathrm{P} 7: 100-200 \mathrm{MeV}$ \\
& $\mathrm{P} 8: 200-400 \mathrm{MeV}$ \\
& $30-400 \mathrm{MeV}, \mathrm{summation}$ of P5, P6, P7 and \\
Opampling time & $\mathrm{P} 8, \mathrm{downlinked} \mathrm{as} \mathrm{housekeeping} \mathrm{parameters}$ \\
& $0.33 \mathrm{~s}$ \\
& continuous working mode \\
& $0.33 \mathrm{~s}$ for $8 \mathrm{energy} \mathrm{channels}$ \\
& $19.8 \mathrm{~s}$ for environment monitoring channel \\
\hline &
\end{tabular}

LEID was calibrated in December 2003 and March 2004 in Centre d'Etude Spatiale des Rayonnements in Toulouse, France. The ground calibration results showed that LEID had a good performance and a similar energy resolution to that of HIA on TC-1.

There are 8 anode sectors distributed within the polar angle range $0^{\circ}-180^{\circ}$, which are numbered Sectors 1 to 8 (from $\left.0^{\circ}-180^{\circ}\right)$. Figure 6 shows the normalized energy response of
Sector 3 at the energy level of $173 \mathrm{eV}$. The analyzer resolution (FWHM) of Sector 3 is about 13\%. Figure 7 shows the intrinsic azimuthal resolution of Sector 3 at the energy level of $173 \mathrm{eV}$. It can be seen from Fig. 7 that the alpha angle resolution (FWHM) of Sector 3 is 5.6. The calibration results also show that the energy response and alpha angle resolution are almost independent of the sectors. The full energy sweep with 16 contiguous energy channels is performed 16 times per spin. Thus, the azimuthal angle resolution is $22.5^{\circ}$.

The scientific objectives of LEID are as follows:

- To study the transport process and acceleration mechanism of upflowing ions in the auroral ionosphere;

- To study the transport process of upflowing ions via the cleft from the ionosphere to both the high-latitude magnetopause boundary layer and the magnetopause boundary layer on the dayside;

- To study the transport process of magnetosheath ions into the ionosphere and plasma sheet through the cleft and the high-latitude magnetopause boundary layer;

- To study the transport process of polar wind ions into the magnetosphere.

The instrument data summary of LEID is given in Table 5 .

\section{First results of HEED, HEPD and HID of TC-1}

TC-1 was launched on 30 December 2003. After the commissioning phase, HEED, HEPD and HID were powered on at 12:30 BLT (Beijing Local Time) on 3 January 2004, and powered off at 17:30. On 5 January, the three Chinese instruments were powered on again and they have operated continuously since then.

In the commissioning phase, the housekeeping data showed that all three instruments work normally. However, the scientific data of HEED and HEPD are abnormal. The scientific data of the 9 energy channels of HEED in the central regions of the inner and outer radiation belts are saturated due to contamination by protons. The scientific data from the 8 energy channels of HEPD are abnormal. The ground data treatment cannot solve these problems. Thus, for HEED, only data prior to saturation are useable. For HEPD, only data from the proton monitoring channel (30$400 \mathrm{MeV}$ ), which are downlinked through the housekeeping data channel, are useable.

\subsection{First results of HEED of TC-1}

Figure 8 shows the high energy electron flux in energy channels E0, E2 and E3 on 18 February 2004. It can be seen that the high energy electron flux decreased rapidly with increasing energy. The high energy electron flux in the energy band E2 is almost identical to that in the energy channel E3 up to $42000 \mathrm{~km}$. Considering that the width of the energy band of $\mathrm{E} 2$ is only half of the width of E3, it also shows that the differential flux indeed decreases with energy. When the satellite is inside the geosynchronous orbit $(\mathrm{H}<36000 \mathrm{~km})$, HEED is saturated. The high energy electron flux decreases instead of 
Table 4. Instrument data summary of HID.

\begin{tabular}{|c|c|}
\hline Quantity & Value \\
\hline Mass & $2.0 \mathrm{~kg}$ \\
\hline Power & $1.7 \mathrm{~W}$ \\
\hline Geometric Factor & $0.255 \mathrm{~cm}^{2} \mathrm{sr}$ \\
\hline Opening Angle (full width) & $\begin{array}{l}60^{\circ} \text {, circular, with a pointing direction perpendicular to the } \\
\text { spin axis of satellite }\end{array}$ \\
\hline Energy range: & $12 \mathrm{MeV}\left(\mathrm{He}^{+}\right)-8 \mathrm{GeV}(\mathrm{Fe})$ \\
\hline Scientific telemetry rate & $0.08 \mathrm{kbit} / \mathrm{s}$ \\
\hline Temperature & $+5^{\circ} \mathrm{C}$ to $+25^{\circ} \mathrm{C}$ \\
\hline Size & $150 \times 120 \times 170 \mathrm{~mm}$ \\
\hline \multirow[t]{11}{*}{ Energy channels } & $\begin{array}{l}\mathrm{He} \text { (four channels): } 12-50 \mathrm{MeV}, 50-130 \mathrm{MeV}, 130-220 \\
\mathrm{MeV}, 220-350 \mathrm{MeV}\end{array}$ \\
\hline & Li: $23-250 \mathrm{MeV}$ \\
\hline & Be: $36-390 \mathrm{MeV}$ \\
\hline & B: $53-640 \mathrm{MeV}$ \\
\hline & C: $70-700 \mathrm{MeV}$ \\
\hline & $\mathrm{N}: 89-890 \mathrm{MeV}$ \\
\hline & O: $109-1090 \mathrm{MeV}$ \\
\hline & F: $137-1341 \mathrm{MeV}$ \\
\hline & Mg: $200-2000 \mathrm{MeV}$ \\
\hline & Ar: $380-400 \mathrm{MeV}$ \\
\hline & Fe: $640-7000 \mathrm{MeV}$ \\
\hline 1 environment monitoring channel & $30-400 \mathrm{MeV}$ \\
\hline Operation mode & continuous working mode \\
\hline Time resolution & $1 \mathrm{~s}$ for Helium ions and $10 \mathrm{~s}$ for other heavy ions \\
\hline
\end{tabular}

increasing. Thus, only the data of HEED outside the geosynchronous orbit is reliable. Between $42000 \mathrm{~km}$ and $43000 \mathrm{~km}$ the lines for E2 and E3 begin to separate. The housekeeping data shows that the instrument was not saturated around that time. So, the farther from the Earth, the more rapidly the high energy flux decreases with the increase in energy.

The designed count range of HEED can reach $10^{5} / \mathrm{s}$, which is 5 times larger than the model predicted peak values in the radiation belt. Thus, it was not the high energy electrons that saturated HEED. A malfunction analysis showed that this abnormality might be due to proton contamination. It was found that the threshold value of a coincidence and anticoincidence circuit was somewhat low. HEED adopts coincidence and anti-coincidence circuit technology to remove the influence of high energy protons. If the threshold value is too low, many high energy protons will be counted as electrons. In this case, the sensor will be saturated and cannot work correctly.

Figure 9 shows the temporal variation of the high energy electron flux at E0 $(0.2-0.4 \mathrm{MeV})$ during the time interval 05:40:00-05:40:20 on 3 January 2004. At 05:40:00 the satellite is at the position $(2.45,6.73,1.23) R_{E}$ in GSE coordinates, outside the center of the outer radiation belt. In each spin period, HEED makes about 12 measurements. The relevant azimuthal angle resolution is about $30 \mathrm{deg}$. In Fig. 9, the high energy electron flux has periodic variations, which are caused by the spin of the satellite. It can be deduced from the amplitude of the pitch angle variation that the angle between the magnetic field and the spin axis was about $16 \mathrm{deg}$. Thus, at this time, the spin of the satellite limited

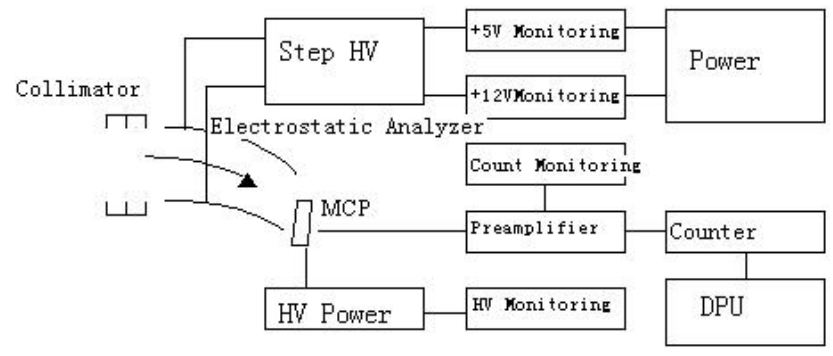

Fig. 5. Block diagram of LEID.

HEED measurements in the pitch angle range $74^{\circ}-106^{\circ}$. The periodic variations of electron flux show that the high energy electrons are still quasi-trapped electrons. Since the satellite is outside the geosynchronous orbit, the periodic variation signatures (trapped particle signatures) become weaker and weaker, and almost disappear at the end of the time interval.

\subsection{First results of HEPD of TC-1}

During the commissioning phase of HEPD on TC-1, the housekeeping data showed that HEPD works normally. The temperatures of HEPD were maintained within the range of $15^{\circ}-25^{\circ}$ and the secondary power values of HEPD were also within normal ranges. However, the data from the 8 energy channels were abnormal and the counts were much smaller than the AP8 model predicted. We have tried to solve this problem through ground calibration work, but the data quality is still not very good. 


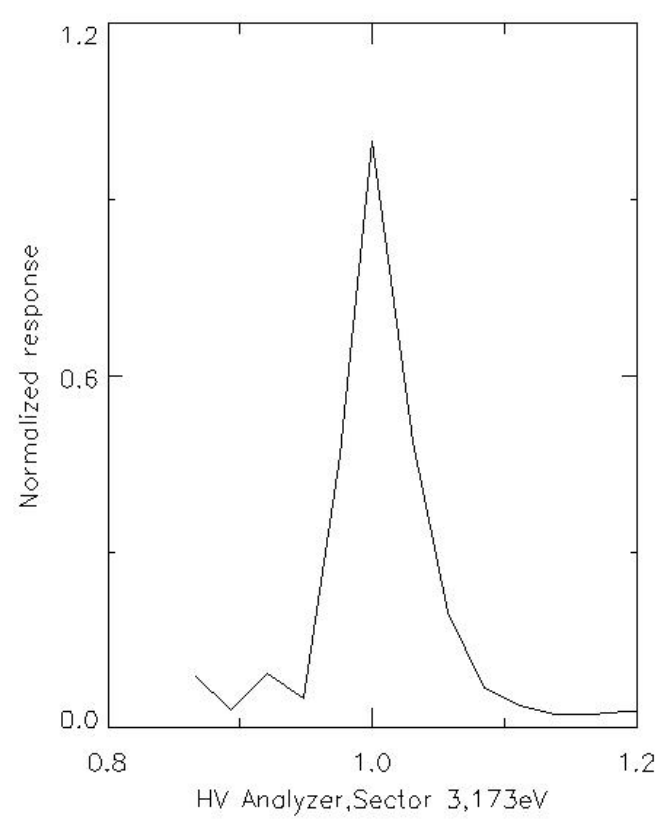

Fig. 6. Normalized energy response of Sector 3 at energy level of $173 \mathrm{eV}$.

For the instrument HEPD, there is another monitoring channel which indicates high energy proton counts in the range 30-400 MeV. This monitoring channel was designed with the purpose of monitoring the working status of HEPD and its data are downlinked through the transfer line for housekeeping data. Figure 10 shows a comparison between AP8-predicted high energy proton omni-directional flux and the directional flux of HEPD/TC-1 on 14 January 2004. The data of HEPD come from the monitoring channel. The peak position and the altitude dependence are consistent with those predicted by the AP8-model.

\subsection{First results of HID of TC-1}

Figure 11 shows the flux of Helium ions in the energy channels He1 and He2 on 15 January 2004. The Helium ion flux in the energy range $12-50 \mathrm{MeV}$ (He1) is much higher than the Helium ion flux in the range $50-130 \mathrm{MeV}$ ( $\mathrm{He} 2)$. Both flux peaks are in the central region of the inner radiation belt. The peak position of the Helium ion flux at higher energy is closer to the Earth than the peak position of the Helium ion flux at lower energy. In addition, the Helium ions in the energy channel He1 (12-50 MeV) are measured over a larger height range than the Helium ions in the energy channel $\mathrm{He} 2$ (50-130 MeV).

\section{First results of HEED, HEPD, HID and LEID of TC-2}

The spacecraft TC-2 of the DSP mission was successfully launched on 25 July 2004. The commissioning of the instruments HEED, HEPD, HID and LFEW started on 28 July. LEID started its commissioning on 1 August 2004.

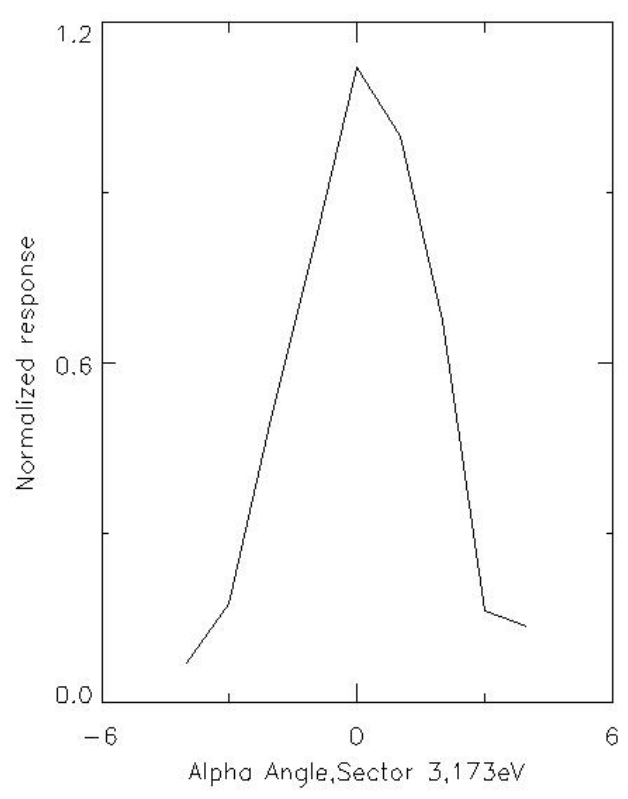

Fig. 7. Intrinsic azimuthal angle resolution of Sector 3 at energy level of $173 \mathrm{eV}$.

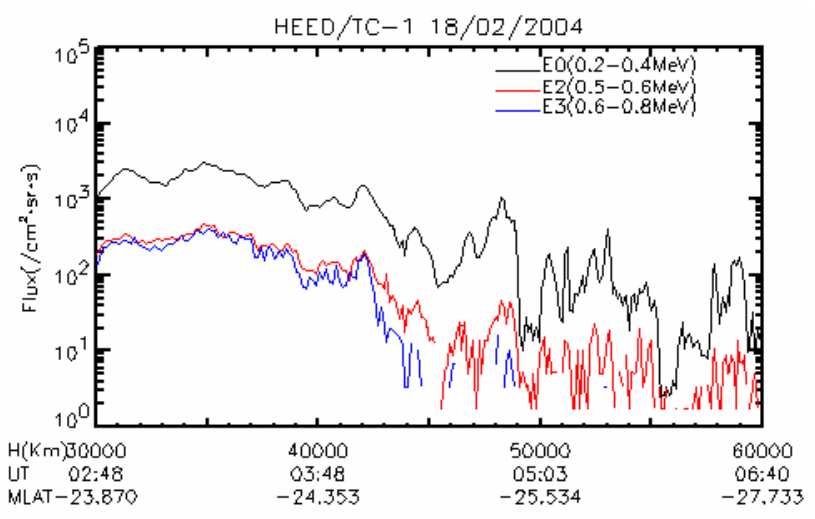

Fig. 8. The minute-averaged high energy electron flux in energy channels E0, E2 and E3 observed by HEED/TC-1 on 18 February 2004.

\subsection{HEED of TC-2}

The design of HEED on TC-2 was slightly modified according to the experience gained from HEED on TC-1. The threshold values are increased and thus the proton contamination can be effectively avoided. Thus, the in-flight performance of HEED on TC-2 was significantly improved. However, HEED was still saturated at some parts of the orbit, mainly near the perigee of TC-2. The downlinked housekeeping data showed that HEED was in good condition after the launch. The temperature of the instrument during the commissioning was kept at $15-25^{\circ}$. The secondary power voltage was stable. The monitoring voltages were shown to be stable in a steady-state test and were a little lower than 
Table 5. Instrument data summary of LEID.

\begin{tabular}{ll}
\hline \multicolumn{1}{c}{ Quantity } & \multicolumn{1}{c}{ Value } \\
\hline Mass & $3.92 \mathrm{~kg}$ \\
Power & $2.9 \mathrm{~W}$ \\
Geometric Factor & $8.1 \times 10^{-4} \mathrm{E}\left(\mathrm{cm}^{2} \mathrm{sr}\right)$ \\
Polar angle resolution & $22.5^{\circ}(8 \mathrm{sectors})$ \\
Azimuthal angle resolution: & 22.5 \\
Measurement range: & $50 \mathrm{eV}-25 \mathrm{keV}$ \\
Scientific telemetry rate & $3 \mathrm{kbit} / \mathrm{s}$ \\
Temperature & $+5^{\circ} \mathrm{C}$ to $+25^{\circ} \mathrm{C}$ \\
Size & Electronic box $300 \times 150 \times 130 \mathrm{~mm}$ \\
& Sensor $125 \times 115 \times 130 \mathrm{~mm}$ \\
Energy channels & $50,75.7115,173,262,396,601,909,1375,2081,3150$, \\
& $4767,7214,10916,16520,25000(\mathrm{eV})$ \\
Energy resolution $\Delta \mathrm{E} / \mathrm{E}$ & $13 \%$ \\
Operation mode & continuous working mode \\
Time resolution & One spin period $(4 \mathrm{~s})$ every three spin periods $(12 \mathrm{~s})$ \\
\hline
\end{tabular}

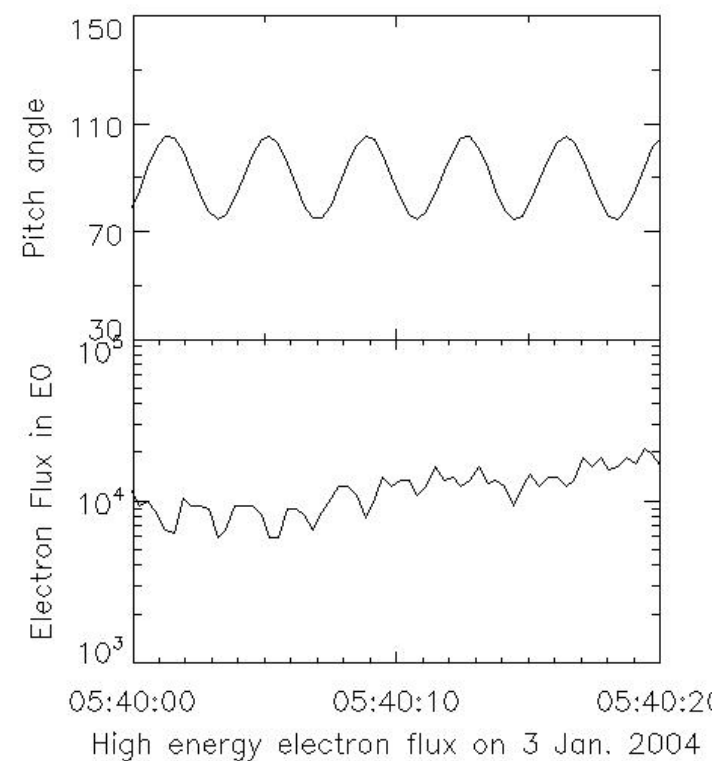

Fig. 9. Temporal variation of the high energy electron flux at E0 (0.2-0.4 MeV) during the time interval 05:40:00-05:40:20 on 3 January 2004.

that recorded during ground tests. This means that the noise level in space is lower than that experienced on the ground.

Figure 12 shows the high energy electron fluxes recorded in energy channels E0, E3 and E6 on 12 August 2004. Here, negative values of height denote the inbound height of the satellite and positive height values denote the outbound height. The fluxes reached their maximum inside the radiation belt, and decreased by about one order of magnitude outside the radiation belt. The fluxes in the energy range $0.2-$ $0.4 \mathrm{MeV}$ were much larger than those in the energy range $0.6-0.8 \mathrm{MeV}$. In the outer radiation belt, similar to the Helium ions, the peak position of the electron flux at higher energy is closer to the Earth than the peak position of the

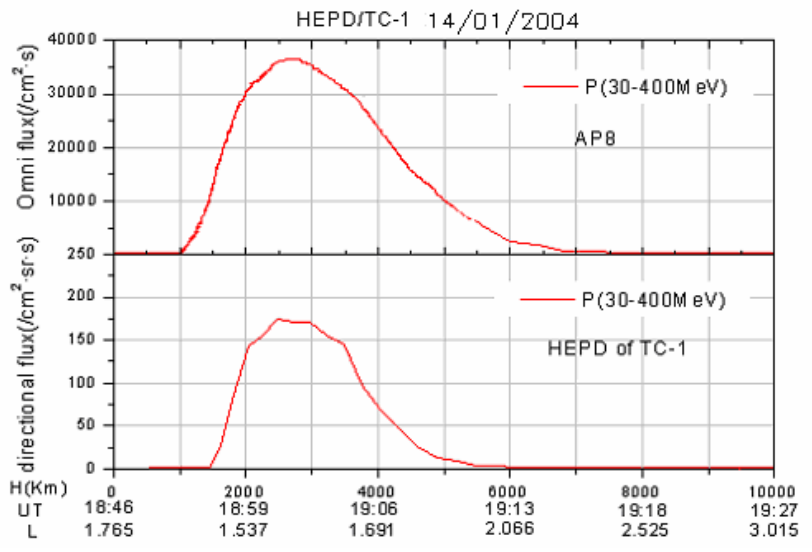

Fig. 10. Comparison between AP8-predicted high energy proton omni-flux and the directional flux of HEPD/TC-1 on 14 January 2004.

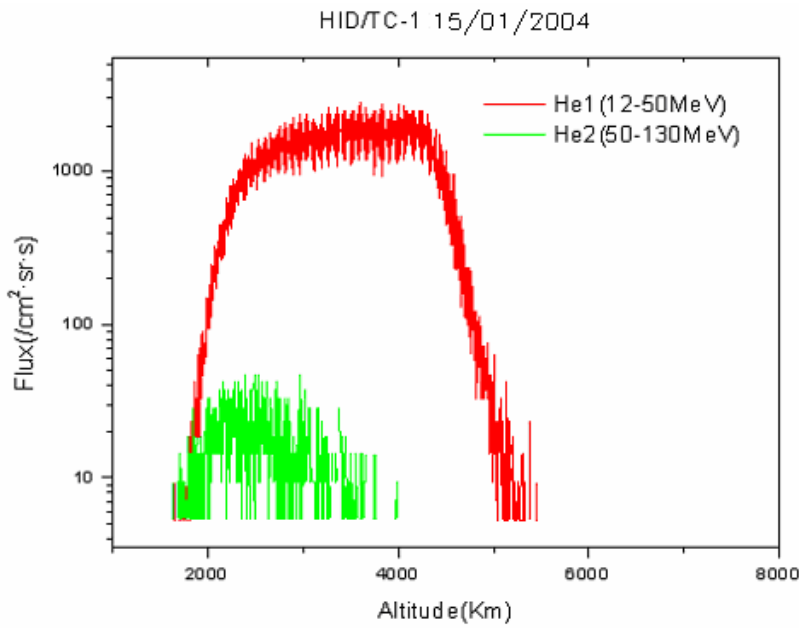

Fig. 11. Flux of Helium ions in energy channels $\mathrm{He} 1$ and $\mathrm{He} 2$ on 15 January 2004. 


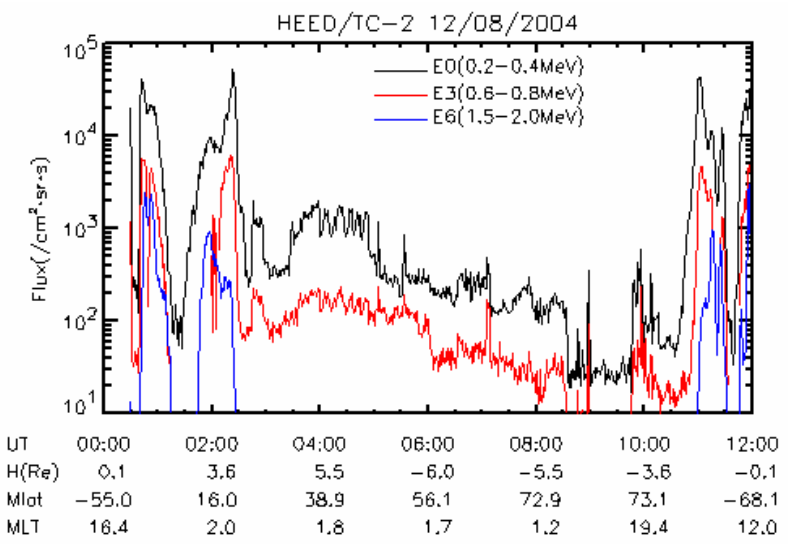

Fig. 12. The minute-averaged high energy electron fluxes recorded in energy channels E0, E3 and E6 on 12 August 2004.

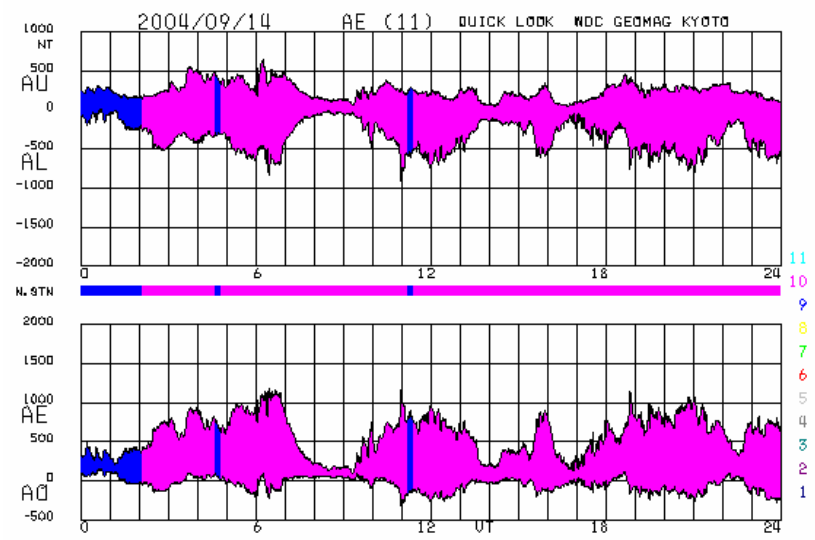

Fig. 13. Substorm $A_{U}, A_{L}$ and $A_{E}$ index recorded on 14 September 2004.

electron flux at lower energy. The high energy electrons in energy channel E0 can exist in the whole orbit, while the high energy electrons in energy channel E6 can only exist in the center regions of the radiation belt.

Since the launch, TC-2 has experienced the arrival of two large Coronal Mass Ejections (CME). One of them occurred on 12 September 2004. This event caused strong disturbances in the magnetosphere. Figure 13 shows the substorm $A_{U}, A_{L}$ and $A_{E}$ indices recorded on 14 September 2004. It can be seen that, within one day, four large substorms occurred. HEED, HEPD and HID recorded the high energy particle response to this composite event.

Figure 14 shows the $D_{s t}$ index and high energy electron flux $(0.2-1.0 \mathrm{MeV}$ and $1-10 \mathrm{MeV})$ observed by HEED on 14 September 2004. It can be seen that the high energy electron flux corresponds relatively well to the $A_{E}$ and $D_{S T}$ indices. The first two peaks in the high energy electron record (at 06:00 and 11:00 UT) occurred when the absolute values of the $D_{S T}$ index and the $A_{E}$ index have reached their maximum values. Although the third peak did not correspond to the peak in the $A_{E}$ and $D_{s t}$ indices, it occurred at the

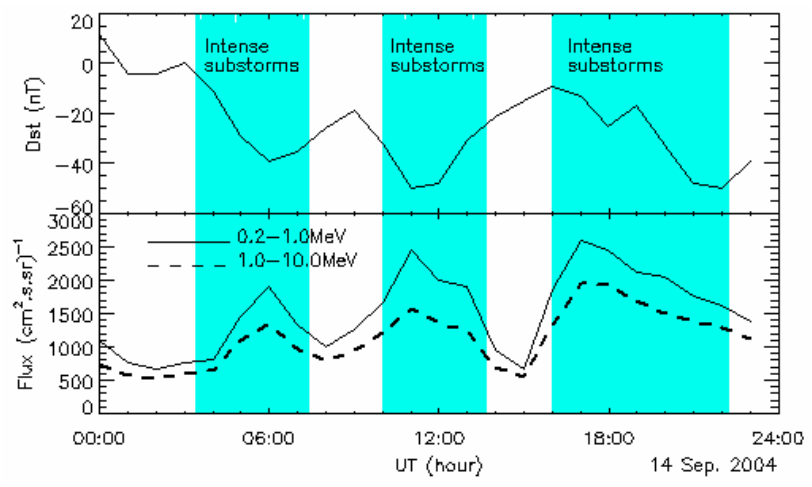

Fig. 14. The $D_{S T}$ index and high energy electron flux $(0.2-1.0 \mathrm{MeV}$ and $1-10 \mathrm{MeV}$ ) observed by HEED/TC-2 on 14 September 2004.

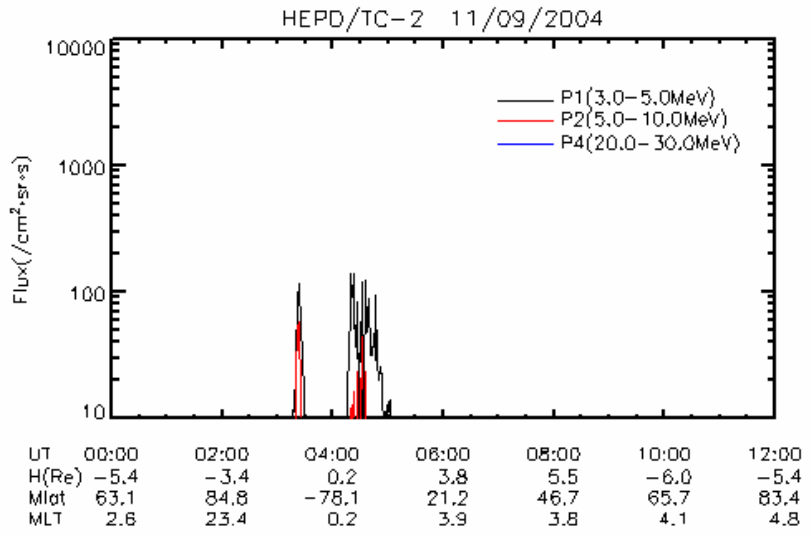

Fig. 15. The minute-averaged high energy proton flux in energy channels P1, P2 and P4 on 11 September 2004.

beginning of the expansion phase of the substorm and originated from substorm-injected energetic electrons as expected by some theoretical works (Li et al., 2005b).

\subsection{HEPD of TC-2}

HEPD was powered on at 11:30 UT on 27 July 2004. The housekeeping data showed that HEPD was in good condition after the launch and it works nominally.

Figure 15 shows the high energy proton flux in energy channels P1, P2 and P4 on 11 September 2004. The absolute values of the $D_{s t}$ index and the $A_{E}$ index are very low, indicating that the magnetosphere was quiet on 11 September 2004. As in the case of the high energy electron fluxes, the peaks of the high energy proton fluxes are found in the central regions of the inner and outer radiation belts. The flux in the inner belt is smaller than that in the outer radiation belt. Outside the radiation belts, the high energy proton flux is very low.

The high energy proton flux observed by HEPD/TC-2 displayed some high energy proton response to the 


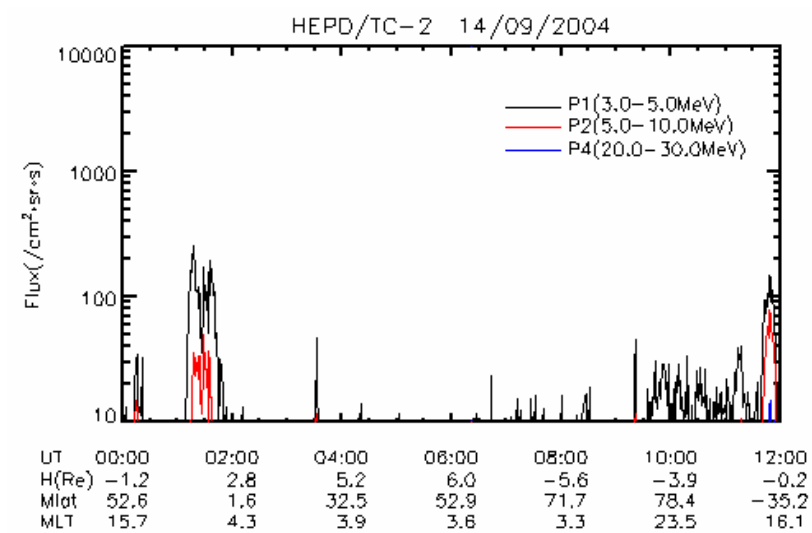

Fig. 16. The minute-averaged high energy proton flux in energy channels P1, P2 and P4 on 14 September 2004.

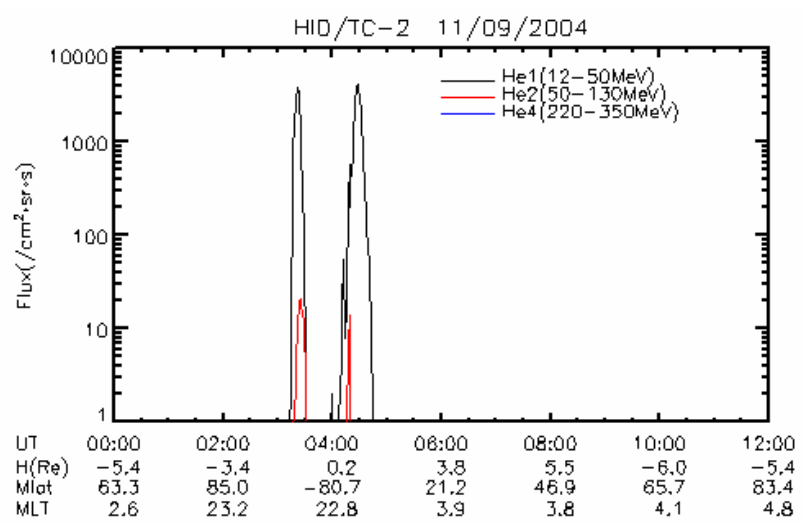

Fig. 17. The minute-averaged helium ion flux in energy channels $\mathrm{He} 1, \mathrm{He} 2$ and $\mathrm{He} 4$ observed by HID on 11 September 2004 (a quiet time).

14 September events. Figure 16 shows the minute averaged proton fluxes in the channels P1, P2 and P4 on 14 September 2004. We compared Fig. 15 (quiet time) and Fig. 16 (active time), and found that the maximum peak flux in the radiation belt increased from around $150 \mathrm{~cm}^{-2} \mathrm{sr}^{-1} \mathrm{~s}^{-1}$ (a quiet time value) to around $280 \mathrm{~cm}^{-2} \mathrm{sr}^{-1} \mathrm{~s}^{-1}$. The increased high energy proton flux was caused directly by the high energy protons associated with the CME. In addition, in contrast to quiet time conditions, the whole orbit traversed by TC- 2 outside the radiation belts was suffused with high energy protons.

\subsection{HID of TC-2}

HID was powered on at 11:30 UT on 27 July 2004. The housekeeping data showed that HID was in good condition after the launch and it works nominally.

Figure 17 shows the fluxes of Helium ions in energy channels $\mathrm{He} 1, \mathrm{He} 2$ and $\mathrm{He} 4$ observed by HID on 11 September 2004. Similar to high energy electrons, the flux of Helium ions decreased rapidly with increasing energy. As in the case

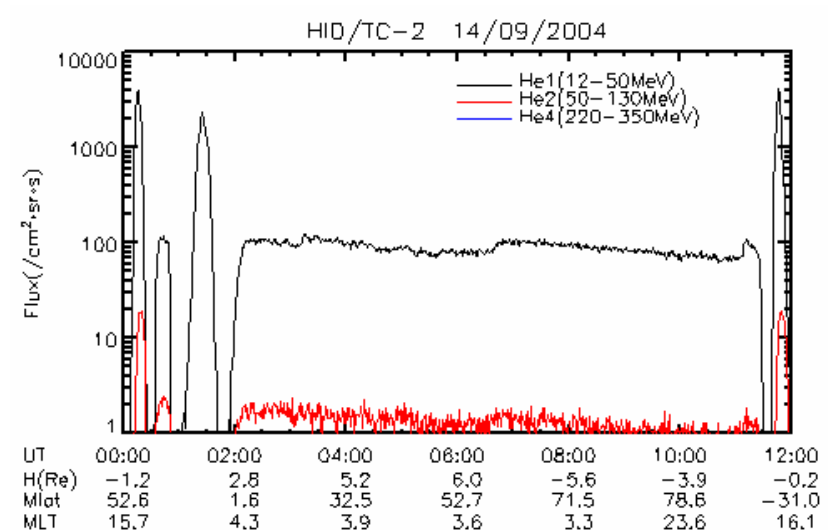

Fig. 18. The minute-averaged helium ion flux in energy channels $\mathrm{He} 1, \mathrm{He} 2$ and $\mathrm{He} 4$ observed by HID on 14 September 2004 (after CME arrival).

of high energy protons, the counts recorded by the instrument were very low outside the radiation belts.

Figure 18 shows the high energy Helium ion flux observed by HID on 14 September 2004. In contrast to the high energy proton measurements, the maximum Helium flux in the radiation belt displayed no obvious change. However, outside the radiation belt, the high energy Helium fluxes increased significantly, and became much larger than the quiet time values. These high energy Helium ions come from the CME. This means that the high energy Helium ion flux from the solar flare was much lower than the quiet flux in the radiation belt and thus could not greatly increase the peak flux value in the radiation belt. However, the high energy helium ion flux from the solar flare was obviously larger than the quiet flux outside the central regions of the radiation belt and could thus fill the whole magnetosphere with high energy Helium ions.

\subsection{LEID of TC-2}

LEID was powered on 9 August 2004. The housekeeping data showed that the instrument was working nominally. However, the scientific data showed that the second sector had almost no data. After 5 September the counts in the first sector also decreased to almost zero and soon had no data.

A preliminary analysis showed that there are two reasons for this malfunction: (1) the sensor performance degraded in space; (2) the electromagnetic interference damaged the pre-amplifier.

With no data in the first and second sectors, we had to use an extrapolation method to calculate the moment quantities (density, temperature and velocity) of the velocity distribution function. Thus these moments are not very precise. The values of Sectors 3 and 4 are used to extrapolate the values of Sector 1 and Sector 2 by means of a simple difference algorithm. Since the first and second polar sectors have very low counts, the calculated plasma density and velocity have 
inherent errors, which change according to the region monitored.

In addition, there is also solar UV contamination in the data when LEID faces the Sun. Thus, the low energy ion flux in the anti-sunward direction is higher than the true value. For the above reasons, we have not obtained reliable scientific data from LEID. More work needs to be done in the future.

\section{Conclusions}

This paper briefly presents the Chinese particle instruments, their data status and first results. Although HEED, HEPD and HID are mounted aboard both TC- 1 and TC-2, the designs of HEED and HEPD of TC-2 were updated based on the experience gained from TC- 1 . Thus, the performances of HEED and HEPD on TC-2 are better than those on TC1. Since LEID is the first Chinese low energy ion detector, we lacked experience with such an instrument. The failure of two sectors and UV contamination make the scientific data not very precise. It is hoped that in the future, we can improve the design of these instruments and construct high quality particle detectors.

Acknowledgements. This work is supported by NSFC Grant 40390153 and by the International Collaboration Research Team Program of the Chinese Academy of Sciences. We are very grateful to Center d'Etude Spatiale des Rayonnements of France for their kind help in the calibration of LEID. Their help is also very important for the improvement of the LEID design in the future Chinese missions. The authors also thank the WDC for Geomagnetism, Kyoto and relevant AE stations.

Topical Editor T. Pulkkinen thanks C. M. Carr and another referee for their help in evaluating this paper.

\section{References}

Baker, D. N., Pulkkinen, T. I., Li, X., Kanekal, S. G., Blake, J. B., Selesnick, R. S., Henderson, M. G., Reeves, G. D., Spence, H. E., and Rostoker, G.: Coronal mass ejections, magnetic clouds, and relativistic magnetospheric electron events: ISTP, J. Geophys. Res., 103, 17 279-17 291, 1998.

Cao, J. B., Zhou, G. C., Wang, X. Y., Wang, D. J., and Cai, C. L.: Relationship among the electron event in April-May 1998, the magnetic storm and interplanetary condition, 2002 Western Pacific Geophysics Meeting, AGU, Wellington, New Zealand, 2002.

Li, X., Baker, D. N., Temerin, M., Cayton, T. E., Reeves, E. G. D., Selesnick, R. S., Blake, J. B., Lu, G., Kanekal, S. G., and Singer, H.: Rapid enhancements of relativistic electrons deep in the magnetosphere during May 15, 1997 magnetic storm, J. Geophys. Res., 104, 4467-4476, 1999.

Li, L. Y., Cao, J. B., and Zhou, G. C.: Relation of the relativistic electron flux change and storm/substorm in the geomagnetosphere, Chin. J. Geophys., in press, 2005a.

Li, L. Y., Cao, J. B., and Zhou, G. C.: Combined acceleration of electrons by whistler-mode and compressional ULF Turbulences near the geosynchronous orbit, J. Geophys. Res., doi:10.1029/2004JA010628, 2005b.

Rème, H., Bosqued, J. M., Sauvaud, J. A., Cros, A., et al.: The Cluster Ion Spectrometry (CIS) Experiment, Space Sci. Rev., 79, 303-350, 1997.

Zhou, G. C., Wang, X. Y., Wang, D. J., Cao, J. B., and Cai, C. L.: $\mathrm{MeV}$ electron-flux enhancement events at the geosynchronous orbit in April-May 1998, Chin. J. Geophys., 44, 285-293, 2001.

Zhu, G., Li, B., Wang, S., Liang, J., et al.: The influence of solar particle event on geosynchronous space environment, Chinese Journal of Space Science, 22, 58-64, 2002. 